\title{
Effect of paleopolyploidy and allopolyploidy on gene expression in banana
}

\author{
Alberto Cenci $i^{*}$ (D), Yann Hueber ${ }^{1}$, Yasmin Zorrilla-Fontanesi ${ }^{2}$, Jelle van Wesemael2 ${ }^{2}$ Ewaut Kissel ${ }^{2}$, Marie Gislard ${ }^{3}$, \\ Julie Sardos ${ }^{1}$, Rony Swennen ${ }^{2,4}$, Nicolas Roux $^{1}$, Sebastien Christian Carpentier ${ }^{2,4}$ and Mathieu Rouard ${ }^{1 *}$
}

\begin{abstract}
Background: Bananas (Musa spp.) are an important crop worldwide. Most modern cultivars resulted from a complex polyploidization history that comprised three whole genome duplications (WGDs) shaping the haploid Musa genome, followed by inter- and intra-specific crosses between Musa acuminata and M. balbisiana (A and B genome, respectively). Unresolved hybridizations finally led to banana diversification into several autotriploid (AAA) and allotriploid cultivars (AAB and $A B B$ ). Using transcriptomic data, we investigated the impact of the genome structure on gene expression patterns in roots of 12 different triploid genotypes covering $A A A, A A B$ and $A B B$ subgenome constitutions.

Results: We demonstrate that (i) there are different genome structures, (ii) expression patterns go beyond the predicted genomic groups, and (iii) the proportion of the B genome influences the gene expression.

The presence of the $\mathrm{B}$ genome is associated with a higher expression of genes involved in flavonoid biosynthesis, fatty acid metabolism, amino sugar and nucleotide sugar metabolism and oxidative phosphorylation. There are cultivar-specific chromosome regions with biased B:A gene expression ratios that demonstrate homoeologous exchanges (HE) between A and B sub-genomes. In two cultivars, aneuploidy was detected. We identified 3674 genes with a different expression level between allotriploid and autotriploid with $\sim 57 \%$ having recently duplicated copies (paralogous). We propose a Paralog Inclusive Expression (PIE) analysis that appears to be suitable for genomes still in a downsizing and fractionation process following whole genome duplications. Our approach allows highlighting the genes with a maximum likelihood to affect the plant phenotype.
\end{abstract}

Conclusions: This study on banana is a good case to investigate the effects of alloploidy in crops. We conclude that allopolyploidy triggered changes in the genome structure of a crop and it clearly influences the gene.

Keywords: Banana, Polyploidy, Paralogs, Genome structure, Transcriptomics

\section{Background}

Bananas (Musa spp.), including dessert and cooking types, are monocotyledonous plants of the Zingiberales, one of the major orders of the Commelinidae clade, also comprising Poales (grasses) and Arecales (palms). Bananas are one of the main fruit crops grown worldwide and are critical for food security in many tropical and subtropical countries.

\footnotetext{
* Correspondence: a.cenci@cgiar.org; m.rouard@cgiar.org

${ }^{1}$ Bioversity International, Parc Scientifique Agropolis II, 34397 Montpellier

Cedex 05, France

Full list of author information is available at the end of the article
}

Banana cultivars, which originated from inter- and intra-specific crosses between Musa acuminata and $M$. balbisiana (both $2 \mathrm{n}=2 \mathrm{x}=22$ ), are mainly triploid ( $2 \mathrm{n}$ $=3 x=33$ ), as their genome is composed of three subgenomes, i.e. three sets of 11 chromosomes. Triploidy-induced sterility combined with parthenocarpy resulted in edible fruits without seeds. A first level of cultivar classification is based on the subgenome origin: autotriploid cultivars contain three subgenomes derived from $M$. acuminata (AAA), whereas allotriploid cultivars contain one or two M. balbisiana-derived subgenomes ( $\mathrm{AAB}$ and $\mathrm{ABB}$, respectively).

(c) The Author(s). 2019 Open Access This article is distributed under the terms of the Creative Commons Attribution 4.0 International License (http://creativecommons.org/licenses/by/4.0/), which permits unrestricted use, distribution, and reproduction in any medium, provided you give appropriate credit to the original author(s) and the source, provide a link to the Creative Commons license, and indicate if changes were made. The Creative Commons Public Domain Dedication waiver (http://creativecommons.org/publicdomain/zero/1.0/) applies to the data made available in this article, unless otherwise stated. 
Once triploidy is established in combination with sterility, plant propagation becomes exclusively vegetative. Therefore, differentiation caused by mutation and clonal selection by farmers resulted in new cultivars, which all derived from a common foundation event.

The analysis of the $M$. acuminata reference sequence (A genome) revealed that the ancestral monocot genome underwent three whole genome duplications (WGDs) independent from those that took place in the evolution of other monocot lineages (e.g. Poaceae) [1]. The history of the Musa WGDs was reconstructed by collinearity analysis of duplicated genes which were estimated to comprise one-third of the total number of genes $[1,2]$, while no genome dominance or biased fractionation was detected [2]. Consequently, the banana genome contains a high number of multigene families with abundant recently originated copies (i.e. paralogs) $[1,3]$, with the post-WGD gene fractionation process still likely ongoing as in other plant species [4]. The analysis of a draft sequence of $M$. balbisiana showed that its genome (B genome) has high degree of homology with a nearly identical number of genes and well-conserved gene-rich regions [5]. However, the two species have a different distribution area and have likely evolved, diverging to better fit their respective environment. For example, differences in codon usage have been detected between the two species [6]. These differences could be useful to understand the genomic constitution of allopolyploids banana cultivars.

So far, expression analyses in allopolyploids have been used for comparisons of gene expression between polyploids and progenitors in coffee and soybean [7, 8], detection of expression bias between paralogs in cotton [9], transcriptomes involved in pathogen defense [10] or in biological processes such as meiosis in Brassica [11]. It has also been shown that the coexistence of different genomes in allopolyploid cultivars can induce Homoeologous Exchanges (HE), i.e. recombination between chromosomes contributed by different species taking place when allotriploidy was established. In Brassica napus, HEs have been shown to cause changes on expression dosage affecting the phenotype of allotetraploid plants [12]. In banana, it had been suggested that intergenome translocations were common $[13,14]$ and it has been shown that chromosome pairing between $\mathrm{A}$ and $\mathrm{B}$ chromosomes at meiosis can occur and translocations between A and B genomes has taken place [15]. However, the relationship between genome structure in allotriploidy and gene expression has not been explored.

In this study, we used data sets obtained from root apexes of 12 banana cultivars covering auto and allotriploids (AAA, $\mathrm{AAB}$ and $\mathrm{ABB}$ ). To investigate the potential impact of the Musa B genome on the phenotype, we conducted differential gene expression and performed genome structure analyses taking into account the paleopolyploid nature of the Musa genome.

\section{Results \\ Read mapping and single-nucleotide polymorphism (SNP) calling}

For this study we first used a published RNAseq dataset [16] on roots of three Musa cultivars (two AAA and one $\mathrm{ABB}$ ('Cachaco')) that we re-mapped on the latest version (v2) of the reference $M$. acuminata sequence (A genome). The number of high-quality reads mapped increased from 81 to $92 \%$ by using the new reference, highlighting the improvement on gene annotation in the reference assembly [17]. Subsequently, a second RNAseq dataset was produced with ten cultivars to broaden our observations with a larger representation of allopolyploids including additional five $\mathrm{ABB}$, one $\mathrm{AAB}$ and three AAA genomes in addition to Musa ABB 'Cachaco' (Table 1). In total, we obtained an average of 24 million single-end reads per genotype. Between 13.1 and 52.2 million single-end reads were obtained per biological replicate (23.6 $\mathrm{M}$ on average) and a range of 67.1 to 90.1\% were uniquely mapped (Additional file 1). The read mapping on the A genome remained quantitatively consistent on the autotriploid and allotriploid cultivars and was not significantly affected by the presence of one or two B genome copies (Additional file 1).

These mappings were used to identify SNPs in order to assess their allopolyploid chromosome structure.

\section{Determination of the genomic structure}

Around 178,000 'Cachaco' SNPs distributed along the 11 Musa chromosomes have been used to estimate the frequency of B genome-specific variants. The SNP positions showing polymorphism within the A reference genome were filtered out by comparing sequence data of 'Grande Naine', 'Mbwazirume' and the reference sequence of the Musa A genome [1] (Fig. 1a). Since the SNP variants specific to the 'Cachaco' A subgenome (i.e. not detected in AAA cultivars and A reference genome) are expected to be rare, the remaining 125,000 SNP variants in 'Cachaco' were assumed to originate from its two B subgenomes. When the variant SNP allele frequency distributions before and after filtering were compared, both displayed a bimodal distribution (peaks at $1 / 3$ and $2 / 3$ ), as expected in the presence of three chromosomes, and a histogram bar corresponding to frequency value of 1 (i.e.: 3/3) (Fig. 1b). The reduction in number of SNPs after the filtering varied according to three categories. As expected, the $2 / 3$ peak was less affected by the filtration, supporting the idea that most of these variants are likely B specific (i.e. present in both B subgenomes of 'Cachaco'). The $1 / 3$ peak lost about half of the SNPs 
Table 1 List of banana cultivars selected for the current transcriptomic study including passport data information

\begin{tabular}{|c|c|c|c|c|}
\hline Accession code & Sample name & Group & Subgroup & Geographical origin \\
\hline ITC0643 & Cachaco & $A B B$ & Bluggoe & \\
\hline ITC0767 & Dole & ABB & Bluggoe & \\
\hline ITC0101 & Fougamou1 & $A B B$ & Pisang Awak & Gabon \\
\hline ITC0652 & Kluai Tiparot & $A B B$ & Klue Teparod & Thailand \\
\hline ITC1483 & Monthan & $A B B$ & Monthan & India \\
\hline ITC0123 & Simili Radjah & $A B B$ & Peyan & India \\
\hline ITC1441 & Pisang Ceylan & $A A B$ & Mysore & \\
\hline ITC1122 & Gros Michel & AAA & Gros Michel & \\
\hline ITC1482 & Poyo & AAA & Cavendish & \\
\hline ITC0575 & Red Dacca & AAA & Red & \\
\hline ITC0180 & Grande Naine & AAA & Cavendish & \\
\hline ITC0084 & Mbwazirume & AAA & $\mathrm{EHAB}$ & Burundi \\
\hline
\end{tabular}

(corresponding to A variants different from the one in the reference A subgenome). The remaining SNPs were, therefore, assumed to be composed of variants specific to one of the two 'Cachaco' B subgenomes and of mutations accumulated during the vegetative propagation in one of the three subgenomes. Finally, the $3 / 3$ peak lost about $60 \%$ of the SNPs, mostly corresponding to the A reference sequence specific SNPs.

The genome constitution of 'Cachaco' being ABB, the $\mathrm{B}$ variant frequency distribution along the $11 \mathrm{Musa}$ chromosomes was around $67 \%$. However, in two interstitial regions, the frequency of $\mathrm{B}$ variants was close to $33 \%$ (chromosomes 4 and 9) and in three other large terminal regions, the frequency of $B$ variants was $100 \%$ for almost all the SNPs (B3:A0 pattern, in chromosome 4 and at both ends of chromosome 11) (Fig. 2; Additional file 2). In these regions, residual SNPs were observed having a bimodal distribution, with peaks at 33 and $67 \%$ (B1:A2 pattern). This indicates the presence of three

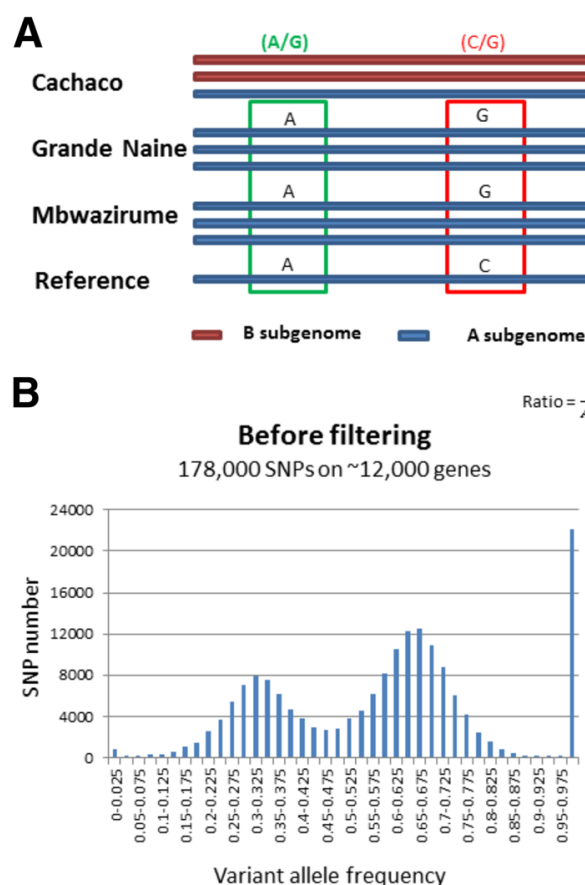

G/T

$(\mathrm{A} / \mathrm{T})$

$\mathrm{T}+\sqrt{(\mathrm{C} / \mathrm{T})}$ $\mathrm{T}$

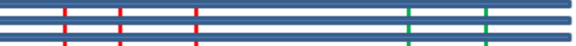

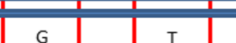

\begin{tabular}{|c|c|c|}
\hline & $T$ & \\
\hline
\end{tabular}

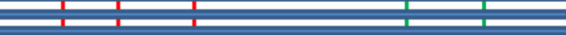
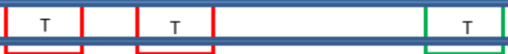

ALT dopth

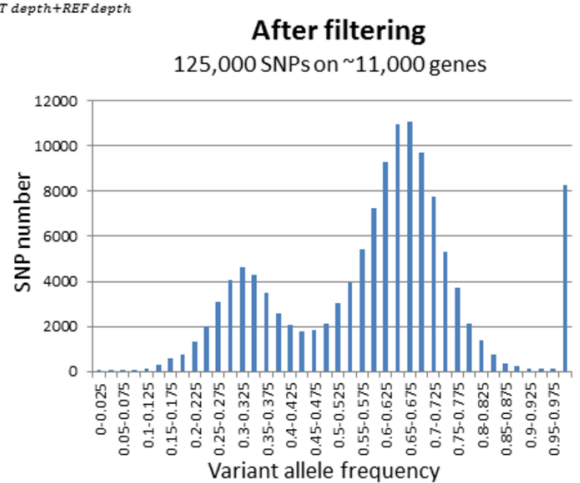

Fig. 1 Filtration method to retrieve B specific alleles in ABB cultivars. a) Concept of the filtration method to keep only polymorphisms from the B subgenomes. In green the retained SNPS, in red the filtered ones. b) Allele frequency distribution of the alternative allele before and after filtration 


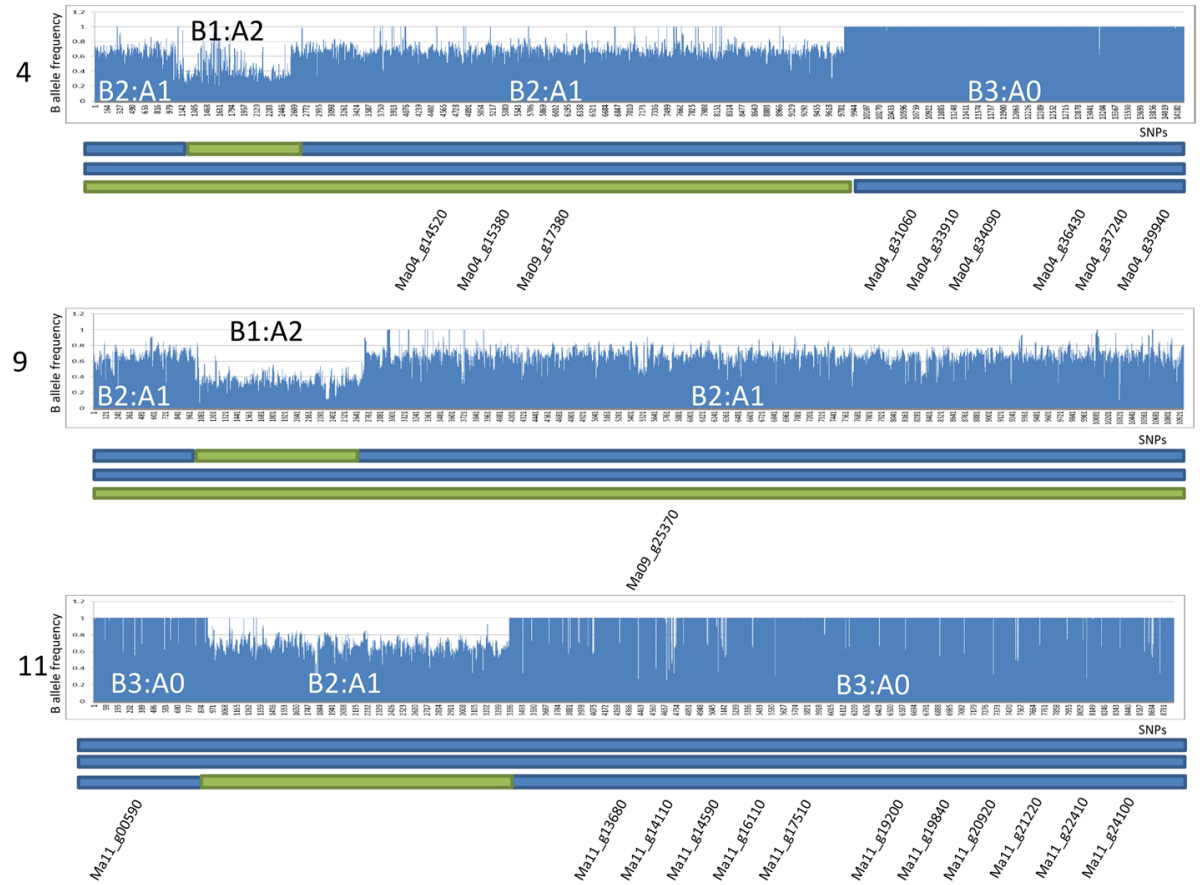

Fig. 2 Detected recombination in chromosomes 4, 9 and 11 of Musa ABB Bluggoe 'Cachaco'. B allele frequency (Y axis) of SNPs ordered according to their position on respective chromosome ( $X$ axis) reveals regions deviating from the expected 2/3 (B2:A1) genomic ratio. Recombinant structure inferred (green and blue segment represent A and B subgenomes, respectively) and distribution of DEGs between 'Cachaco' and 'Grande Naine'/'Mbwazirume' along the chromosomes

genomes, excluding the hypothesis of partial A subgenome deletion and, thus, suggesting HE due to recombination. Among the 33,615 genes annotated in the Musa chromosomes, 3105 (9.24\%) genes of 'Cachaco' do not have any A subgenome homeoallele while 861 (2.56\%) have a double dose of the A homeoallele.

The same approach was applied to all allotriploid cultivars (Additional file 2). 'Monthan' and 'Dole' shared all the recombinations detected in 'Cachaco'; 'Simili Radjah' shared some recombination with the 'Cachaco', 'Dole' and 'Monthan' recombination pattern (Additional file 2) and showed an additional interstitial region on chromosome 5, where the genome ratio is B1:A2. 'Fougamou1' showed several recombination events, but all were different from those of 'Cachaco', 'Dole' and 'Monthan'. 'Kluai Tiparot' also had several recombinations different from the ones detected in 'Cachaco', 'Dole' and 'Monthan' and 'Fougamou1'. However, in 'Kluai Tiparot', all recombinations were characterized by a B3:A0 ratio. Finally, in the single $\mathrm{AAB}$ cultivar 'Pisang Ceylan', some recombinations with both $\mathrm{B} 0: \mathrm{A} 3$ and $\mathrm{B} 2: \mathrm{A} 1$ ratios were detected (Additional file 2).

In two cultivars, aneuploidy was also detected: the whole chromosome 8 is missing in 'Dole' and the long arm of 'Simili Radjah' chromosome 5 has only two of the three expected allelic doses.

\section{B genome impact on the transcriptomes of allotriploid cultivars}

A Partial Least Square (PLS) analysis clearly distinguished $\mathrm{ABB}$ from AAA cultivars, whereas the unique $\mathrm{AAB}$ cultivar 'Pisang Ceylan' had an intermediate position (Fig. 3).

Genes with a higher expression in the cultivars containing the $\mathrm{B}$ genome were enriched in the following pathways: flavonoid biosynthesis, fatty acid metabolism, amino sugar and nucleotide sugar metabolisms and oxidative phosphorylation (Fig. 4).

\section{Expression analysis in a paleopolyploid genome}

A set of 3674 genes was found significantly differentially expressed between 'Cachaco' and both AAA cultivars, 'Grande Naine' and 'Mbwazirume' (Additional file 3). About $57 \%$ of the differentially expressed genes (DEGs) were identified as part of multigene families as unique or multiple representatives (Additional file 4). Among these genes, $541(14.73 \%)$ were located in the B3:A0 regions, i.e. they have only the $\mathrm{B}$ genome homeoallele, whereas $33(0.90 \%)$ were in the region with double dose of A homeoalleles.

Due to the high proportion of DEGs belonging to multicopy families, a subset of 54 DEGs was selected for manual curation to gain insight into their potential impact on the phenotype (Additional file 5). One-third of those genes (18) were located in the B3:A0 regions, 


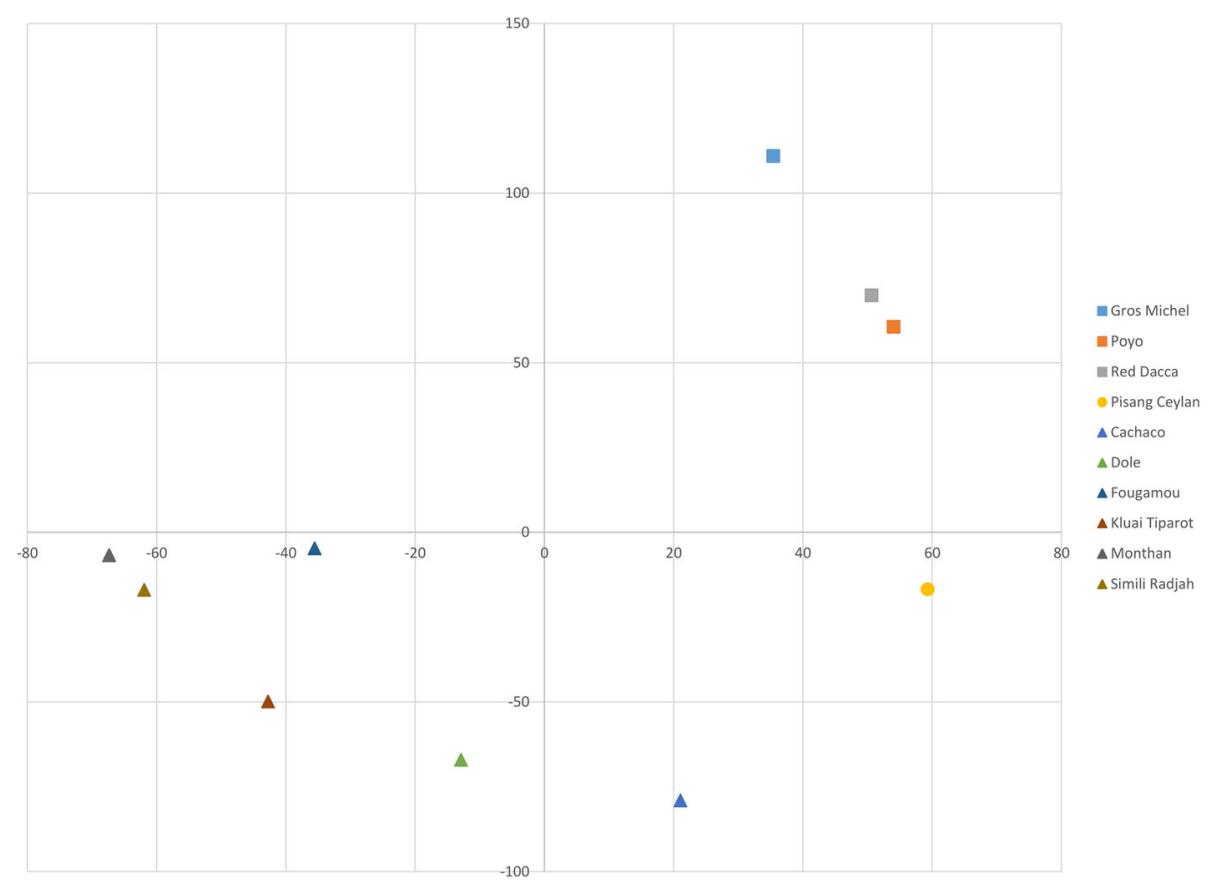

Fig. 3 Partial Least Squares (PLS) analysis on 10 cultivars having auto or allopolyploid genome constitution. Squares, circles and triangles indicate genome constitution of the analysed cultivars (AAA, AAB and ABB, respectively). $X$ and $Y$ axes represent $P C 1$ and $P C 2$ variables respectively
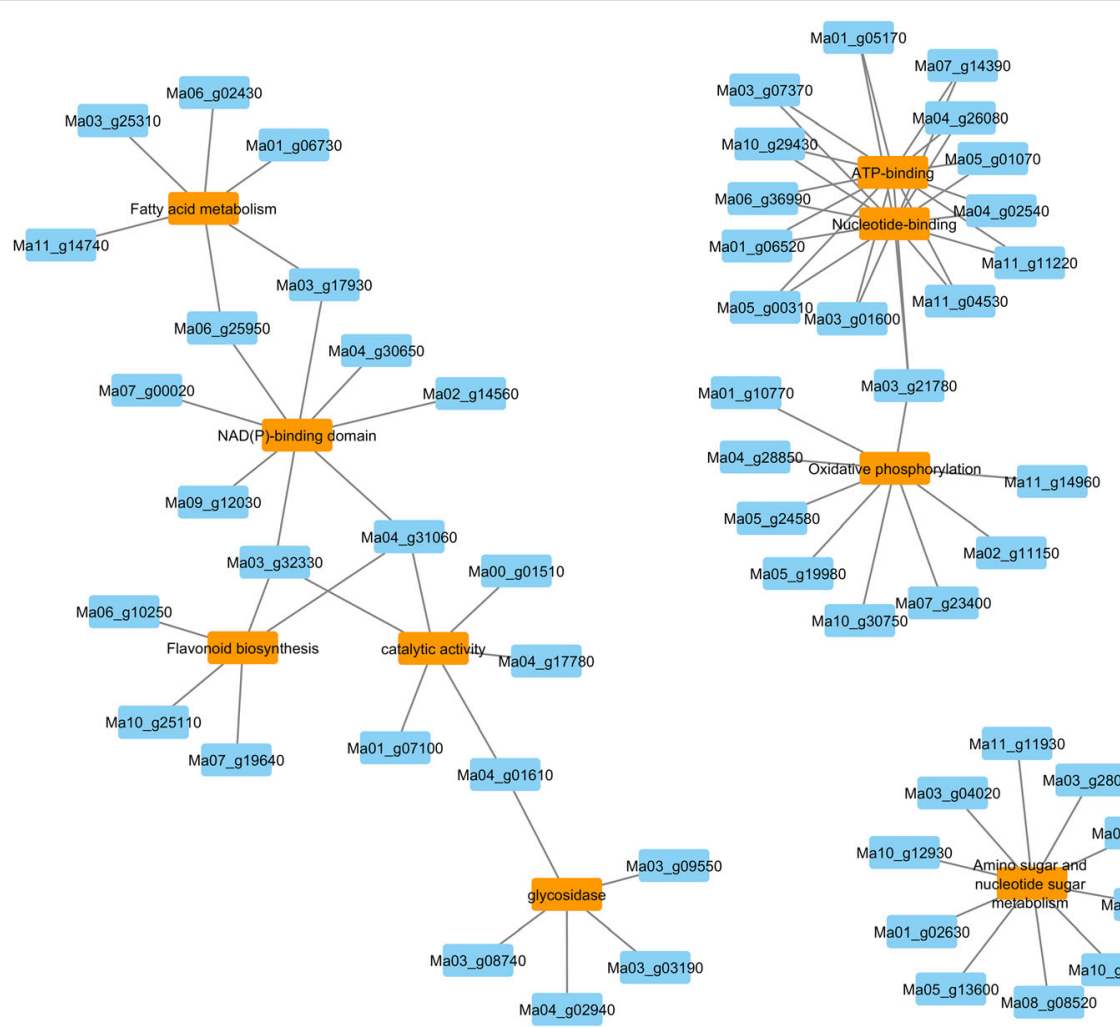

Fig. 4 Pathways identified by enrichment analysis on gene set. Pathways are indicated in orange squares and gene id are represented by the blue squares 
whereas none were located in the B1:A2 regions. Based on paralogous identification, eight belonged to multicopy (>10) tandem repeated clusters and were no longer analyzed. Among the remaining 46 DEGs, 13 did not have any paralog in the Musa genome and 1-9 paralogs were found for the other 34 (Additional file 5). On those 34, a differential expression analysis called Paralog Inclusive Expression (PIE) analysis was performed to take into account the relative expression of the DEG and its lineage specific paralogs. Among them, the expression of only two genes is not overruled by paralogs: 1) Ma04_g36430, whose two of the three found paralogs (being the tandemly duplicated copies Ma04_g36440 and Ma04_g36450) have a consistent expression pattern with the sampled gene (i.e. no expression in 'Cachaco' but expression in both AAA cultivars), whereas no or very low expression was detected for the third paralog (Ma10_g01400) (Fig. 5a); 2) Ma11_g14590 whose unique paralog (Ma00_t02460) has consistent expression with Ma11_g14590 (Fig. 5b). For the remaining 32 DEGs, the paralog expression was globally higher than the sampled gene, overruling or reducing the impact of the expression difference between 'Cachaco' and AAA cultivars (Ma05_g02000 and Ma11_g20920 as example in Fig. 5c and d; Additional file 6). In the larger dataset including six $\mathrm{ABB}$ and three AAA cultivars, differential expression of almost all the 15 validated DEGs, over- or under-expressed in 'Cachaco' vs AAA cultivars, was confirmed to be associated with the presence of the $B$ genome (Fig. 6).

In the second dataset, DEGs were identified between each allopolyploid and each of the three AAA genotypes. For each allopolyploid, DEGs detected in all the three comparisons were selected (Table 2). In 'Pisang Ceylan' $(\mathrm{AAB})$ a lower number of DEGs was detected (131), whereas among the ABB cultivars the detected DEGs spanned between 547 and 1661 (Table 2). Moreover, the DEGs in chromosome regions showing a deviating genome contribution ratio were counted (Table 2) and similarly to 'Cachaco' all the regions with a B3:A0 structure in $\mathrm{ABB}$ genotypes (and with $\mathrm{B} 2 \mathrm{~A} 1$ in $\mathrm{AAB}$ genotype) showed a significant excess of DEGs compared to a random distribution. In the regions where the $\mathrm{B}$ contribution was lower than expected (i.e. B1:A2 and $\mathrm{B} 0: \mathrm{A} 3$ in $\mathrm{ABB}$ and $\mathrm{AAB}$, respectively) the number of DEGs was globally under-represented (Table 2).

\section{Discussion}

Homoeologous exchanges occurred between a and

\section{B genomes}

Polyploidy is known to induce changes in genome structure and gene expression [12] and banana is no

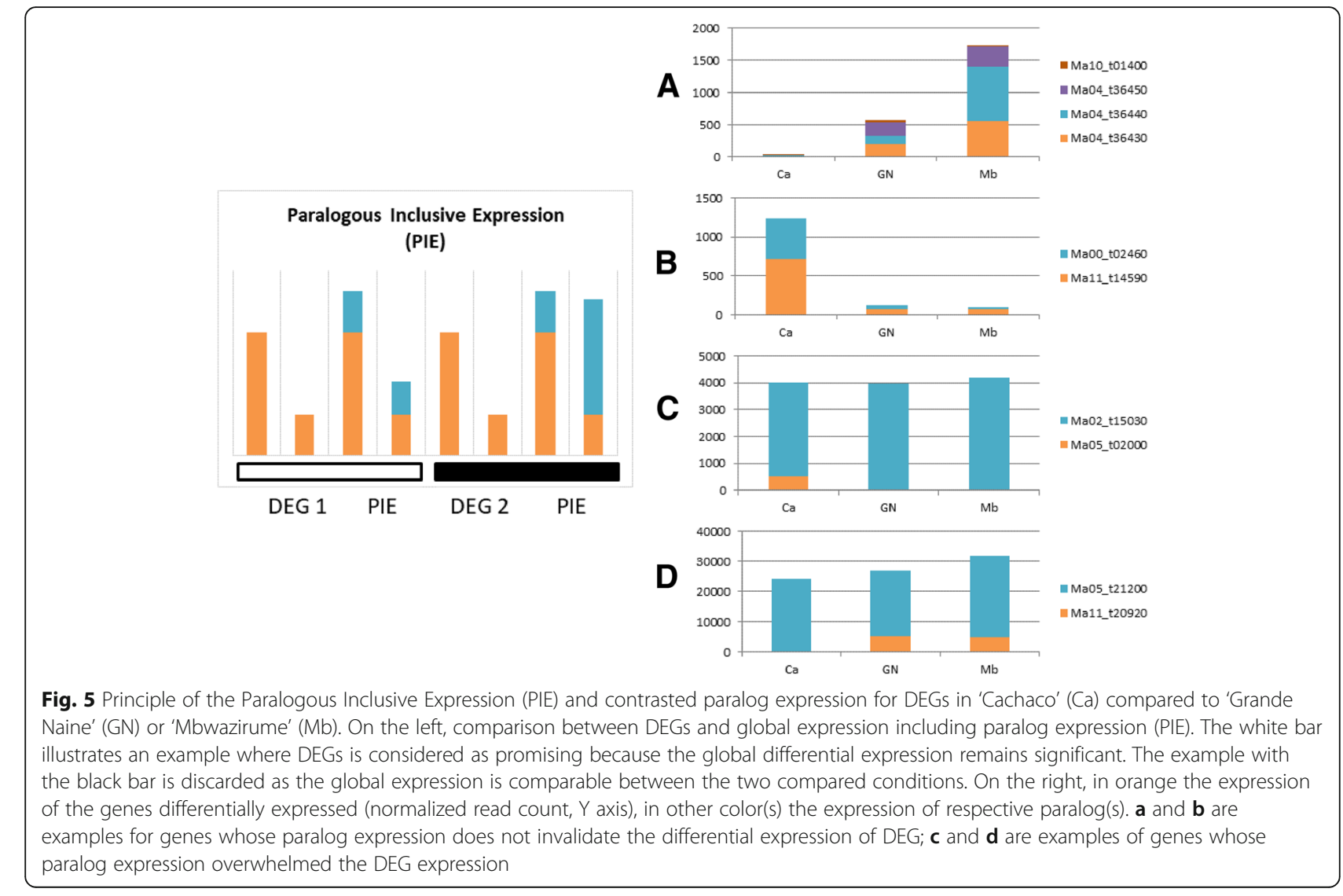




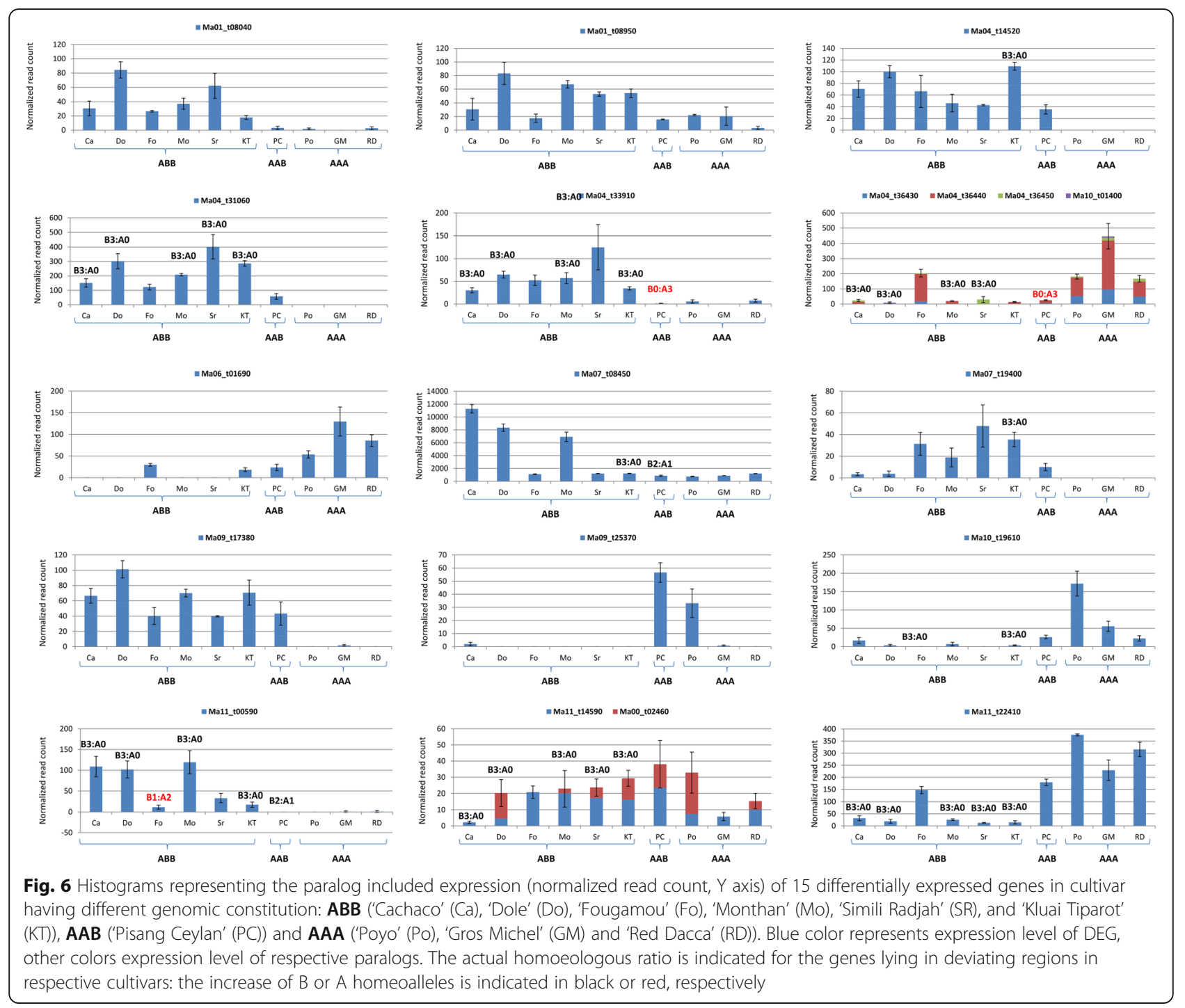

Table 2 Over-representation of DEGs (allo- vs autotriploids) in recombined regions of allotriploids

\begin{tabular}{|c|c|c|c|c|c|c|c|}
\hline & Genome & B3:A0 regions & & & B1:A2 regions & & \\
\hline Genotype & DEGs & Genes & DEGs & $P$ value & Genes & DEGs & $P$ value \\
\hline Cachaco & $987(0.03)$ & $3105(0.09)$ & $168(0.17)$ & $3.0^{e^{-17}}$ & $861(0.03)$ & $12(0.01)$ & $<0.01$ \\
\hline Dole* & $577(0.02)$ & $3105(0.10)$ & $111(0.14)$ & $<0.01$ & $861(0.03)$ & $8(0.01)$ & $<0.01$ \\
\hline Fougamou & $823(0.02)$ & $3483(0.10)$ & $188(0.23)$ & $7.1^{e-32}$ & $1226(0.04)$ & $11(0.01)$ & $<0.01$ \\
\hline Kluai Tiparot & $1661(0.05)$ & $22,105(0.66)$ & $1249(0.75)$ & $5.29^{\mathrm{e}-16}$ & N/A & N/A & N/A \\
\hline Monthan & $558(0.02)$ & $3105(0.09)$ & $102(0.17)$ & $1.11^{\mathrm{e}-11}$ & $861(0.03)$ & $4(0.01)$ & $<0.01$ \\
\hline \multirow[t]{2}{*}{ Simili Radjah ${ }^{a}$} & $547(0.02)$ & $1847(0.06)$ & $97(0.16)$ & $1.05^{\mathrm{e}-25}$ & $393(0.01)$ & $1(0.00)$ & $<0.05$ \\
\hline & Genome & B2:A1 regions & & & B0:A3 regions & & \\
\hline Genotype & DEG & Genes & DEGs & $P$ value & Genes & DEGs & $P$ value \\
\hline Pisang Ceylan & $131(0.004)$ & $3116(0.09)$ & $28(0.21)$ & $2.8^{\mathrm{e}-06}$ & $803(0.02)$ & $2(0.002)$ & Not significant \\
\hline
\end{tabular}

${ }^{a}$ Chromosome 8 and 5 data were excluded from Dole and Simili Radjah, respectively

Gene and DEG numbers (between brackets their ratio with the 35,276 genes annotated in the V2 Musa sequence) were compared in regions with deviating subgenome ratios 
exception [18]. The structure of the allotriploid genomes studied here is not the mere sum of $11 \mathrm{~A}$ chromosomes from $M$. acuminata and $22 \mathrm{~B}$ chromosomes from $M$. balbisiana. The observation of chromosome regions showing deviation from the expected $2 \mathrm{~B}: 1 \mathrm{~A}$ ratio (3B:A0 and $1 \mathrm{~B}: 2 \mathrm{~A}$, according to the region), or, in the case of 'Pisang Ceylan' (AAB) from the expected 1B:2A ratio (2B:1A or $0 \mathrm{~B}: 3 \mathrm{~A}$ ) implies that recombinant events left their tracks in the allotriploid genomes. It was already hypothesized that most, if not all, banana cultivars have genomes consisting of different proportions of A- and B-genome chromosomes and/or recombinant chromosomes based on chromosomal, nuclear and cytoplasmic DNA and protein data [13]; this was also recently reported for a few cultivars [19].

Since the triploid cultivars are sterile and vegetatively-propagated, it is likely that the detected recombinations originated during the formation of the allotriploid genotypes and were fixed by vegetative propagation. Due to their obliged clonal nature and from an evolutionary point of view, the triploid bananas should be considered as hybrid lineages, even if allotriploid bananas share some features with allopolyploid species (e.g. fixed heterozygosity or inter-genomic interactions). The presence of regions with deviating homeoallele contributions could impact the phenotypes, in particular for the regions 3B:0A (or 0B:3A), where the missing $\mathrm{A}$ (or $\mathrm{B}$ ) genome regions induce a localized lack of inter-genomic heterozygosity and highlights the possible expression differences between the A and B subgenomes. For instance, around 9\% of the Musa annotated genes lie in genomic regions where the 'Cachaco' genome ratio is $3 \mathrm{~B}: \mathrm{A} 0$, but, when only the DEGs between 'Cachaco' and both AAA cultivars were considered (3674 genes), the 3B:A0 gene fraction is significantly higher $\left(14.7 \%, p<2.1^{2 \mathrm{e}-36}\right.$ in first dataset and results reported in Table 2 for the second one). We can conclude that the substitution of A homeoalleles increases the probability of significant changes in gene expression and, consequently, in phenotype. Similar dosage-dependent effects on expression of genes in genomic regions that underwent homoeologous exchanges were already observed in Brassica napus [12].

\section{Expression differences between Allo- and autotriploid cultivars are mainly influenced by the presence/absence of the $B$ genome}

We aimed to understand the impact of the B subgenome presence on the total gene expression in allopolyploids. The general expression level is altered between the 3 genomic groups (Fig. 3). This confirms the impact of the $\mathrm{B}$ genome presence on the gene expression. The presence of the B genome in the roots leads to a higher expression of genes involved in pathways like flavonoid biosynthesis, fatty acid metabolism, amino sugar and nucleotide sugar metabolism and oxidative phosphorylation. The biological functions of flavonoids are linked to their potential cytotoxicity and their capacity to interact with enzymes through protein complexation. Some flavonoids provide stress protection, for example, acting as scavengers of free radicals such as reactive oxygen species (ROS), as well as chelating metals that generate ROS via the Fenton reaction [20]. Roots are for their energy completely dependent on the carbohydrates they receive from the source organs. The preferred way to generate the energy is via metabolizing carbohydrates and fatty acids via oxidative phosphorylation. Fatty acid metabolism, ATP binding, catalytic properties and amino and nucleotide sugar metabolism are general important pathways for root growth \& development.

See the wood for the trees: the role of paralogous genes Banana has a complex paleopolyploid genome with multiple paralogs. Three WGDs were inferred in the evolutionary history of the banana (haploid) genome. The two more recent and "almost simultaneous" WGDs ( $\alpha$ and $\beta$ ) have left more than one-third of genes in multiple copies [1, 2]. WGDs are common and recurrent evolutionary phenomena in plants $[21,22]$ and evolution by gene duplication is understood to be an important source of phenotypic novelties [23]. For instance, neo-functionalization (i.e. functional divergence of gene copies) or sub-functionalization (space-temporal repartition of the gene function) could give evolutionary advantages. However, at genomic scale, this duplicated status is unstable and tends to evolve towards a diploid status by structural recombinations and by a genome downsizing or fractionation (i.e. loss of duplicated genes that follows the WGDs) $[4,24]$. This fragmentation can be a long process that involves the removal of duplicated and functionally redundant genes by accumulation of mutations in the additional copies. In most cases, those mutations (including partial or total deletion) compromise the function of the coded protein. The high number of duplicated genes in the Musa genome [1], the higher number of Musa gene family members in well-studied gene families when compared to other species $[3,25]$ and the comparative genome wide analysis of duplicated genes in a large sample of angiosperms [26] (Fig. 4), suggests that the fragmentation process in the Musa genome is still ongoing which makes the proportion of paralogs with redundant function likely high.

In our analyses, we found paralogs for the large majority of DEGs selected in ABB/AAA comparisons. The presence of paralogous gene copies (potentially being functionally redundant), makes it trickier to interpret differential gene expression results. Considering an enzymatic function ensured by more than one gene, the 
impact of regulation changes needs to be considered for all the gene copies (Fig. 5).

A panel of genes showing highly significant different expression between 'Cachaco' (ABB) and AAA cultivars was analyzed to identify paralogs and to determine the expression changes and the possible impact on the phenotype by a Paralog Inclusive Expression (PIE) analysis (Additional file 5). In a subset of 46 DEGs between 'Cachaco' and two AAA cultivars, 32 DEGs have paralogs with expression that overwhelms the differences observed in the considered genes, with a likely negligible impact on the phenotype. Fourteen genes could potentially have an influence on the phenotype as 13 of them have no paralogs (Additional file 5) and the other two have paralogs whose expression level is very reduced or consistent with the analyzed DEGs. Interestingly, these genes were selected among the most significant DEGs and were detected in almost all $\mathrm{ABB}$ genotypes.

For some genes, these copies may be still diverging in their expression after the M. acuminata and M. balbisiana lineage separation. Indeed, in a given genome a gene can have recently lost (or strongly limited) its expression without consequences for the phenotype due to the presence of redundant paralogs, and be maintained in the other genome, inducing a significant different expression between A and B homeoalleles but with no obvious effect on the phenotype. However, the impact of several enriched genes in the same pathway or function gives confidence of their potential effect on the phenotype.

\section{Conclusions}

We observed marked differences between auto and allotriploid transcriptomes, the most significant DEGs often being correlated with the presence or absence of the $B$ subgenome. There is a large variability among the $A B B$ cultivars due to the different genomic structures detected in the sampled cultivars (homoeologous exchanges), which testify independent foundation events for the ABB subgroup and, likely, with different A and B genome contributions.

Since the occurrence of WGDs is one of most frequent evolutionary events in plants, the occurrence of paralogs could be generalized for all plant species. However, the impact of duplicated and functionally redundant genes is inversely correlated with the time elapsed from the most recent WGD and with the specific evolution rate. In fact, the progress of the fractionation and the neo- and sub-functionalization of duplicated genes reduce the occurrence of functionally redundant genes and, consequently, increase the probability that an observed expression difference impacts the phenotype.

The presence of multi-copy genes adds to the background noise in whole genome expression analysis, thus increasing the need for expert analyses to draw correct conclusions. For species having genomes with a high number of paralogs, an automatic pipeline for paralogous detection combined with the evaluation of regulation changes in gene multi-copy context would be relevant.

\section{Methods}

\section{Plant material and growth conditions}

Twelve varieties belonging to $\mathrm{AAA}, \mathrm{AAB}$ and $\mathrm{ABB}$ subgroups were supplied by the Bioversity International Musa Germplasm Transit Centre (ITC), hosted at KU Leuven, Belgium (Table 1). Plants were grown as described by Zorrilla-Fontanesi et al. (2016), using a Bronson incubator (Bronson Incubator Services B.V., Nieuwkuijk, Netherlands). In vitro plants were grown for 21 days, after which root tips $(4-5 \mathrm{~cm}$ long) were collected and snap frozen separately in liquid nitrogen and stored at $-80^{\circ} \mathrm{C}$.

\section{RNA extraction and CDNA library sequencing}

Total RNA was extracted as described in [27]. For cDNA library construction, RNA integrity was checked by the ExperionTM (BIO-RAD Laboratories, Inc. USA; RQI > 9.4) and BioAnalyzer (Agilent; RIN > 7.8). In total, 9 and 30 RNA samples (for the first and second experiment, respectively) were isolated from 3 biological replicates per genotype. Multiplex sequencing on an Illumina HiSeq2000 was performed as $100 \mathrm{bp}$, single reads at the Montpellier GenomiX facility as described in Zorrilla-Fontanesi et al. (2016).

\section{RNA-Seq filtering, mapping and SNP calling}

RNA-Seq reads were quality-filtered using the Illumina purity filter. Quality of reads was checked using FastQC v0.11.2 [28]. Reads were cleaned to remove adapter sequences and low-quality ends (phred score $>20$ ) with Cutadapt v2.7.9 (Martin, 2011). After trimming, reads inferior to $30 \mathrm{bp}$ were discarded. Reads were then aligned against the reference $M$. acuminata genome (DH Pahang) v2 $[17,29]$ using the splice junction mapper for RNA-Seq STAR v2.5.0 [30] in a 2-pass mode with default parameters. Read groups were added for each alignment file (SAM). Reads were then split using SplitNCigarReads and locally realigned with IndelRealigner from GATK (Genome Analysis ToolKit) v3.4 [31]. SNPs were called on uniquely mapped reads with UnifiedGenotyper from GATK (ploidy parameter set to 3). The variant annotation and effect prediction was performed with SnpEff v4.1 [32]. An overview of the bioinformatics pipeline is shown in Additional file 7. 


\section{Allotriploid genomic structure}

Our dataset of genotypes 'Mbwazirume,' 'Cachaco' and 'Grande Naine' was used as a starting point for further analysis. All the reads were realigned to the newest version of the Musa genome (derived from the genomic sequence of $M$. acuminata DH Pahang v2 (Martin et al., 2016)).

The allotriploid genomic structure was verified by checking the $2 / 3$ expected contribution of $B$ homeoallele variants ( $1 / 3$ for 'Pisang Ceylan', AAB) of SNPs in the expressed genes along the 11 chromosomes. In order to reduce the bias introduced by SNPs due to the A sequence polymorphism, only SNP positions monomorphic in available A genomes (DH Pahang and RNA-Seq data of AAA cultivars 'Grande Naine' and 'Mbwazirume') were taken into account. At each retained SNP position, the alternative nucleotide to the reference variant was considered the $\mathrm{B}$ genome variant (Fig. 1a).

\section{Multivariate analysis}

To find genotype specific gene expression patterns, a Partial Least Squared analysis was performed with the NIPALS algorithm on the normalized read counts (Statistica 13, Non Sigma) of the larger sample dataset. 35,307 annotated genes were considered as $\mathrm{X}$ variables and the number of $\mathrm{B}$ chromosomes as $\mathrm{Y}$ variables $(0,1,2)$. Normalized reads were subjected to ANOVA analysis and Benjamini correction (33,667 variables).

\section{Gene enrichment}

To analyse possible gene enrichment, the Benjamini corrected variables were selected according to their $\mathrm{ABB}$ or AAA profile and their accession numbers were converted to entrez gene IDs using the conversion tool (http://banana-genome-hub.southgreen.fr/convert).

Entrez gene IDs were submitted to DAVID [33]. Significant enrichments (EASE threshold 0.1) were exported and visualized using Cytoscape [34].

\section{Differential gene expression analyses}

The number of reads in genes were counted with HTSeq-count [35] using the corresponding gene annotation file and the "union" mode. Differential gene expression was evaluated using edgeR v3.12.0 [36] in the $R$ statistical environment ( $\mathrm{R}$ Core Team, 2013). The $p$-value was adjusted for multiple testing by controlling the false discovery rate (FDR) at $\leq 5 \%$. Data were normalized using RLE [37].

A subset of DEGs was created for manual curation processes. We first sorted all DEGs based on their respective p-value for 'Cachaco' vs 'Mbwazirume' and 'Cachaco' vs 'Grande Naine' $(n=3)$ (Additional file 3). Then, we considered arbitrarily the DEGs in the first
150 positions of both rankings to select the most highly differently expressed genes common to both.

\section{Paralog detection and paralog inclusive expression (PIE) analysis}

Identification of Musa specific paralogs on the whole set of differentially expressed genes (DEGs) was performed using Orthofinder v2.2.1 with protein sequences from Musa acuminata v2, Oryza sativa v7, Arabidopsis thaliana $v 10$ and from Vitis vinifera $v 1$. The number of paralogs was defined by counting the number of gene occurrence of Musa within each orthogroup.

The presence of lineage specific paralogs (in-paralogs) in the Musa genome for a limited number of selected DEGs was verified by BLASTp analysis on Non-redundant protein database at NCBI [38]. Musa genes, having higher scores than genes from any other species, were considered in-paralogs. Even if orthology/paralogy is defined based on phylogenetic analysis, we considered our simplified approach based on sequence similarity appropriate to find paralogs and the possible bias due to lack of identification of paralogs with high sequence divergence as negligible. The same approach was used in [39]). When one or more paralogs were found, normalized counting of each paralog was added to the one of the respective DEG and paralog inclusive expression was compared among samples.

\section{Additional files}

Additional file 1: Sequencing and genome mapping statistics for the 12 genotypes considered in the study. Since 'Cachaco' was present in both the experiments, it was represented twice. (PNG $1026 \mathrm{~kb}$ )

Additional file 2: Chromosome intervals (defined on genes coordinates) showing B:A ratio deviating from the expected genomic constitution. CDM indicates the three cultivars sharing the same pattern of deviating regions ('Cachaco', 'Dole' and 'Monthan'). (DOCX 16 kb)

Additional file 3: List of 3674 genes showing different expression levels between 'Cachaco' (ABB) and both AAA cultivars ('Grande Naine' and 'Mbwazirume'). LogFC, logCPM, $p$-value and FDR are reported for both comparisons. (XLSX $513 \mathrm{~kb}$ )

Additional file 4: Distribution of the 3674 DEGs by number of paralogs. (PNG $128 \mathrm{~kb}$ )

Additional file 5: Sample of genes differentially expressed in 'Cachaco' (ABB) compared to AAA cultivars ('Mbwazirume' and 'Grande Naine'). (DOCX $18 \mathrm{~kb}$ )

Additional file 6: Histograms representing the paralog included expression (normalized read count, $Y$ axis) for 58 genes having significant higher expression in 'Cachaco' (Ca) than in 'Grande Naine' (GN) and 'Mbwazirume' (Mb). Blue color represents expression level of DEG, other colors expression level of respective paralogs. (PPTX $146 \mathrm{~kb}$ )

Additional file 7: Schematic view of the bioinformatics workflow for differential gene expression and genome structure identification. (PNG 310 kb)

\section{Abbreviations}

DEG: Differentially expressed gene; HE: Homoeologous exchange;

PIE: Paralog Inclusive Expression; PLS: Partial Least Square; ROS: Reactive oxygen species; SNP: Single-nucleotide polymorphism; WGD: Whole genome duplication 


\section{Acknowledgements}

The authors would like to thank Hien Do and Edwige Andre (KU Leuven) for technical assistance and to Rachel Chase for careful English editing.

\section{Funding}

This work was supported by the Bioversity International project 'Adding value to the ITC collection through molecular and phenotypic characterization', financed by the Belgian Development Cooperation and by donors through their contributions to the CGIAR Fund (http://www.cgiar.org/ our-funders/), and in particular to the CGIAR Research Program, Roots, Tubers and Bananas. The funders had no role in study design, data collection and analysis, interpretation of results, decision to publish, or preparation of the manuscript.

\section{Availability of data and materials}

The datasets generated and analysed during the current study are available in the Sequence Read Archive (SRA) of the National Center for Biotechnology Information (NCBI) repository, https:/www.ncbi.nlm.nih.gov/bioproject/ PRJNA305241.

\section{Authors' contributions}

AC, SCC, and MR designed the study. NR, RS supervised the study. JVW, YZ-F, EK and MG generated the data. AC, YH, JS, SCC, MR, analyzed the data. AC, SCC and MR wrote the manuscript. All Co-authors read and reviewed the manuscript. All authors read and approved the final manuscript.

\section{Ethics approval and consent to participate}

Not applicable.

\section{Consent for publication}

Not applicable.

\section{Competing interests}

The authors declare that they have no competing interests.

\section{Publisher's Note}

Springer Nature remains neutral with regard to jurisdictional claims in published maps and institutional affiliations.

\section{Author details \\ 'Bioversity International, Parc Scientifique Agropolis II, 34397 Montpellier Cedex 05, France. ${ }^{2}$ Laboratory of Tropical Crop Improvement, Division of Crop Biotechnics, KU Leuven, B-3001 Leuven, Belgium. ${ }^{3}$ MGX-Montpellier GenomiX, Montpellier Genomics and Bioinformatics Facility, F-34396 Montpellier, France. ${ }^{4}$ Bioversity International, Willem De Croylaan 42, B-3001 Leuven, Belgium. International Institute of Tropical Agriculture. c/o The Nelson Mandela African Institution for Science and Technology (NM-AIST), P.O. Box 447, Arusha, Tanzania.}

\section{Received: 26 October 2018 Accepted: 18 March 2019}

Published online: 27 March 2019

\section{References}

1. D'Hont A, Denoeud F, Aury J-M, Baurens F-C, Carreel F, Garsmeur O, et al. The banana (Musa acuminata) genome and the evolution of monocotyledonous plants. Nature. 2012;488:213-7

2. Garsmeur O, Schnable JC, Almeida A, Jourda C, D'Hont A, Freeling M. Two evolutionarily distinct classes of Paleopolyploidy. Mol Biol Evol. 2014;31:448-54.

3. Cenci A, Guignon V, Roux N, Rouard M. Genomic analysis of NAC transcription factors in banana (Musa acuminata) and definition of NAC orthologous groups for monocots and dicots. Plant Mol Biol. 2014;85(1-2):63-80.

4. Wendel JF. The wondrous cycles of polyploidy in plants. Am J Bot. 2015:102:1753-6.

5. Davey MW, Gudimella R, Harikrishna JA, Sin LW, Khalid N, Keulemans J. A draft Musa balbisiana genome sequence for molecular genetics in polyploid, inter- and intra-specific Musa hybrids. BMC Genomics. 2013;14:683.

6. Kundapura Venkataramana R, Hastantram Sampangi-Ramaiah M, Ajitha R, Khadke GN, Chellam V. Insights into Musa balbisiana and Musa acuminata species divergence and development of genic microsatellites by transcriptomics approach. Plant Gene. 2015;4:78-82.
7. Ilut DC, Coate JE, Luciano AK, Owens TG, May GD, Farmer A, et al. A comparative transcriptomic study of an allotetraploid and its diploid progenitors illustrates the unique advantages and challenges of RNA-seq in plant species. Am J Bot. 2012:99:383-96.

8. Combes M-C, Dereeper A, Severac D, Bertrand B, Lashermes P. Contribution of subgenomes to the transcriptome and their intertwined regulation in the allopolyploid Coffea arabica grown at contrasted temperatures. New Phytol. 2013;200:251-60.

9. Adams KL, Cronn R, Percifield R, Wendel JF. Genes duplicated by polyploidy show unequal contributions to the transcriptome and organ-specific reciprocal silencing. Proc Natl Acad Sci. 2003;100:4649-54.

10. Powell JJ, Fitzgerald TL, Stiller J, Berkman PJ, Gardiner DM, Manners JM, et al. The defence-associated transcriptome of hexaploid wheat displays homoeolog expression and induction bias. Plant Biotechnol J. 2017;15:533-43.

11. Braynen J, Yang Y, Wei F, Cao G, Shi G, Tian B, et al. Transcriptome analysis of floral buds deciphered an irregular course of meiosis in Polyploid Brassica rapa. Front Plant Sci. 2017;8. https://doi.org/10.3389/ fpls.2017.00768.

12. Lloyd A, Blary A, Charif D, Charpentier C, Tran J, Balzergue S, et al. Homoeologous exchanges cause extensive dosage-dependent gene expression changes in an allopolyploid crop. New Phytol. 2017. https://doi. org/10.1111/nph.14836.

13. De Langhe E, Hřibová E, Carpentier S, Doležel J, Swennen R. Did backcrossing contribute to the origin of hybrid edible bananas? Ann Bot. 2010;106:849-57.

14. Carpentier SC, Panis B, Renaut J, Samyn B, Vertommen A, Vanhove A-C, et al. The use of 2D-electrophoresis and de novo sequencing to characterize inter- and intra-cultivar protein polymorphisms in an allopolyploid crop. Phytochemistry. 2011;72:1243-50

15. Noumbissié GB, Chabannes M, Bakry F, Ricci S, Cardi C, Njembele J-C, et al. Chromosome segregation in an allotetraploid banana hybrid (AAAB) suggests a translocation between the $\mathrm{a}$ and $\mathrm{B}$ genomes and results in eBSV-free offsprings. Mol Breed. 2016;36:38.

16. Zorrilla-Fontanesi Y, Rouard M, Cenci A, Kissel E, Do H, Dubois E, et al. Differential root transcriptomics in a polyploid non-model crop: the importance of respiration during osmotic stress. Sci Rep. 2016;6:22583.

17. Martin G, Baurens F-C, Droc G, Rouard M, Cenci A, Kilian A, et al. Improvement of the banana "Musa acuminata" reference sequence using NGS data and semi-automated bioinformatics methods. BMC Genomics. 2016:17:243.

18. van Wesemael J, Hueber Y, Kissel E, Campos N, Swennen R, Carpentier S. Homeolog expression analysis in an allotriploid non-model crop via integration of transcriptomics and proteomics. Sci Rep. 2018;8:1353.

19. Baurens F-C, Martin G, Hervouet C, Salmon F, Yohomé D, Ricci S, et al. Recombination and large structural variations shape interspecific edible bananas genomes. Mol Biol Evol. 2019;36:97-111.

20. Winkel-Shirley B. Biosynthesis of flavonoids and effects of stress. Curr Opin Plant Biol. 2002;5:218-23.

21. Van de Peer $Y$, Fawcett JA, Proost $S$, Sterck L, Vandepoele $K$. The flowering world: a tale of duplications. Trends Plant Sci. 2009;14:680-8.

22. Van de Peer $Y$, Mizrachi E, Marchal K. The evolutionary significance of polyploidy. Nat Rev Genet. 2017;18:411-24.

23. Flagel LE, Wendel JF. Gene duplication and evolutionary novelty in plants. New Phytol. 2009;183:557-64.

24. Murat F, Armero A, Pont C, Klopp C, Salse J. Reconstructing the genome of the most recent common ancestor of flowering plants. Nat Genet. 2017:49:490-6.

25. Cenci A, Rouard M. Evolutionary analyses of GRAS transcription factors in angiosperms. Front Plant Sci. 2017;8. https://doi.org/10.3389/fpls.2017.00273.

26. Li Z, Defoort J, Tasdighian S, Maere S, de PY, Smet RD. Gene duplicability of Core genes is highly consistent across all angiosperms. Plant Cell. 2016:28:326-44

27. Podevin N, Krauss A, Henry I, Swennen R, Remy S. Selection and validation of reference genes for quantitative RT-PCR expression studies of the nonmodel crop Musa. Mol Breed:1-16.

28. Andrews S. Babraham Bioinformatics-FastQC a quality control tool for high throughput sequence data; 2015.

29. Droc G, Lariviere D, Guignon V, Yahiaoui N, This D, Garsmeur O, et al. The Banana genome hub. Database. 2013;2013:bat035-bat035.

30. Dobin A, Davis CA, Schlesinger F, Drenkow J, Zaleski C, Jha S, et al. STAR: ultrafast universal RNA-seq aligner. Bioinformatics. 2013;29:15-21. 
31. McKenna A, Hanna M, Banks E, Sivachenko A, Cibulskis K, Kernytsky A, et al. The genome analysis Toolkit: a MapReduce framework for analyzing nextgeneration DNA sequencing data. Genome Res. 2010;20:1297-303.

32. Cingolani P, Platts A, Wang LL, Coon M, Nguyen T, Wang L, et al. A program for annotating and predicting the effects of single nucleotide polymorphisms, SnpEff: SNPs in the genome of Drosophila melanogaster strain $\mathrm{w}^{1118}$; iso-2; iso-3. Fly (Austin). 2012;6:80-92.

33. Huang DW, Sherman BT, Lempicki RA. Systematic and integrative analysis of large gene lists using DAVID bioinformatics resources. Nat Protoc. 2009:4:44-57.

34. Shannon P, Markiel A, Ozier O, Baliga NS, Wang JT, Ramage D, et al. Cytoscape: a software environment for integrated models of biomolecular interaction networks. Genome Res. 2003;13:2498-504.

35. Anders S, Pyl PT, Huber W. HTSeq--a Python framework to work with highthroughput sequencing data. Bioinformatics. 2015;31:166-9.

36. Robinson MD, McCarthy DJ, Smyth GK. edgeR: a Bioconductor package for differential expression analysis of digital gene expression data. Bioinformatics. 2010;26:139-40.

37. Anders S, Huber W. Differential expression analysis for sequence count data. Genome Biol. 2010;11:R106.

38. Pruitt KD, Tatusova T, Brown GR, Maglott DR. NCBI reference sequences (RefSeq): current status, new features and genome annotation policy. Nucleic Acids Res. 2012;40:D130-5.

39. De Smet R, Adams KL, Vandepoele K, Van Montagu MCE, Maere S, Van de Peer $Y$. Convergent gene loss following gene and genome duplications creates single-copy families in flowering plants. Proc Natl Acad Sci. 2013;110:2898-903.

Ready to submit your research? Choose BMC and benefit from:

- fast, convenient online submission

- thorough peer review by experienced researchers in your field

- rapid publication on acceptance

- support for research data, including large and complex data types

- gold Open Access which fosters wider collaboration and increased citations

- maximum visibility for your research: over $100 \mathrm{M}$ website views per year

At $\mathrm{BMC}$, research is always in progress.

Learn more biomedcentral.com/submissions 